\title{
Inverted Rib Waveguides Theoretical Analysis of Sensing Properties
}

\author{
C. Tyszkiewicz*
}

Department of Optoelectronics, Silesian University of Technology, Akademicka 2, 44-100 Gliwice, Poland

Optical waveguides are fundamental components of chemical and biochemical evanescent wave sensors. Presented work is devoted to theoretical investigations of homogeneous and surface sensitivity characteristics of the inverted rib waveguide structure. The inverted rib waveguide discussed in this paper is composed of an ion-exchange based channel waveguide formed in a glassy substrate and a uniform, high refractive index, sol-gel derived $\mathrm{SiO}_{2}-$ $\mathrm{TiO}_{2}$ film. Characteristics of a homogeneous sensitivity, a surface sensitivity and fundamental modes sensitivity differences in function of a uniform film thickness and a maximal refractive index contrast of the gradient index channel waveguide are given. The influence of the $\mathrm{SiO}_{2}-\mathrm{TiO}_{2}$ film thickness on sensitivity characteristics is discussed. The analysis is carried out on a bases off the effective index method used with the transfer matrix method.

DOI: $10.12693 /$ APhysPolA.124.582

PACS: 42.79.Gn, 07.07.Df

\section{Introduction}

Planar evanescent wave sensors (PEWS) are widely used in chemical and biological sensing applications. A structure of a typical PEWS sensor is composed of a slab or a rib waveguide exposed to an ambient of which refractive index changes are detected or detected is the presence of the sensitive film covering this waveguide. The key parameters of PEWS are a homogeneous sensitivity and a surface sensitivity defined as derivatives of effective refractive indexes in respect of cover parameters - a refractive index of the ambient and a sensitive film thickness [1]:

$$
\Delta n_{\mathrm{eff}}=\left(\frac{\partial n_{\mathrm{eff}}}{\partial n_{\mathrm{c}}}\right) \Delta n_{\mathrm{c}}+\left(\frac{\partial n_{\mathrm{eff}}}{\partial w_{\mathrm{s}}}\right) \Delta w_{\mathrm{s}}
$$

where $S_{\mathrm{H}}=\mathrm{d} n_{\mathrm{eff}} / \mathrm{d} n_{\mathrm{c}}$ is the homogeneous sensitivity, $S_{\mathrm{D}}=\mathrm{d} n_{\mathrm{eff}} / \mathrm{d} w_{\mathrm{s}}$ is the surface sensitivity, $n_{\text {eff }}$ is an effective refractive index, $n_{\mathrm{c}}$ is a refractive index of the ambient and $w_{\mathrm{s}}$ is a thickness of the sensitive film.

The magnitude of these sensitivities depends solely on the ratio of optical power carried in the ambient or in the sensitive film to the total power carried in the structure. Changes of effective refractive indexes can be measured using grating couplers [2,3] or planar waveguide interferometers [4-6]. The highest sensitivities are achievable for single modal or bimodal uniform waveguides having high refractive index [7-9]. However thicknesses of such waveguides are small, being of the order of wavelength fraction [10, 11]. It is a source of high coupling losses between PEWS and optical fibre. These losses can be reduced by using an inverted, composite rib waveguides. A composite waveguide has two layers of which one is capable of mode guidance only in the presence of the second layer. In papers $[12,13]$ there has been shown that the sensitivity of a gradient homogeneous $(\mathrm{G}-\mathrm{H})$

*e-mail: cuma.tyszkiewicz@polsl.pl composite waveguide based PEWS can be significantly increased when one of two interfering modes is confined to a thin uniform $\mathrm{SiO}_{2}-\mathrm{TiO}_{2}$ film having high refractive index whereas the second mode is carried in an ion-exchange based gradient slab waveguide. Slab waveguides are mainly used for construction of difference interferometers, whose simple construction and high sensitivities gained from $\mathrm{G}-\mathrm{H}$ nature are their undisputable assets. Aforementioned advantages of $\mathrm{G}-\mathrm{H}$ composite structures can be utilized for construction of double-path planar interferometers if a gradient slab waveguide is replaced with a gradient rib waveguide.

This paper is devoted to a theoretical analysis of a relation between the selected parameters of a composite inverted rib waveguides, described in Sect. 2. In the next section there is given the basic insight of the analysis method. Finally in Sect. 4 the results - homogeneous and surface sensitivity and fundamental modes sensitivity difference characteristics are presented.

\section{Composite structure}

Investigated composite planar structure is composed of an ion-exchange based rib waveguide, formed in a soda-lime glassy substrate, on the top of which there is a thin, uniform $\mathrm{SiO}_{2}-\mathrm{TiO}_{2}$ slab film. Refractive index profile (RIP) of the ion-exchange rib waveguide cannot be expressed by some analytical function [14]. However formation of the uniform slab film requires an annealing and after it RIP can be approximated by a following Gaussian function:

$$
n(x, y)=n_{b}+\Delta n \exp \left(-\frac{e^{2} x^{2}+(y-d)^{2}}{g^{2} x^{2}}\right),
$$

where $d$ is a rib profile depth, $n_{b}$ is substrate refractive index, $\Delta n$ is a maximal refractive index contrast. Parameters $g$ and $e$ are introduced to limit profile and its decrease in a spatial domain. The profile width $t$ is related to its depth $d$ by a parameter $e$ in Eq. (2): $t=d / e$. The parameter $g$ is derived from an assumption that $n(\mp t, 0)=n_{b}+\delta n$, where $\delta n$ is a very small increase of RIP at its horizontal boundary $(\delta n \ll \Delta n)$. Uniform 
slab film is characterized by a refractive index $n_{2}$ and a thickness $d_{2}$. The morphological parameters of the investigated composite structure are schematically presented in Fig. 1. Analysis presented in this paper is carried out for a wavelength $\lambda=0.677 \mu \mathrm{m}$.

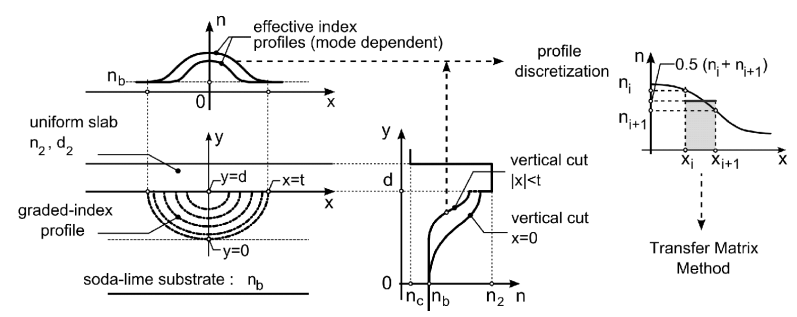

Fig. 1. Diagram of the composite inverted rib structure: $n_{2}, d_{2}$ - refractive index and thickness of the uniform slab film, $d, t$ - rib profile depth and width, $n_{b}, n_{\mathrm{c}}$ - refractive indexes of the substrate and the ambient.

\section{Analysis method}

The analysis of composite, inverted rib waveguides was carried out with the application of the effective index method (EIM) [15] used with the transfer matrix method [16]. The basic assumption of the EIM method is that the electromagnetic field can be expressed with the separation of spatial variables. For the normalized electric field component $E(x, y)$ the solution to the Helmholtz equation is given by

$$
E(x, y)=F_{i}(x) G_{i}(y),
$$

where $i=x, y$ denotes the direction of polarization of these components.

Separation postulated in Eq. (3) narrows down the analysis of the two-dimensional rib waveguide to the analysis of a set of vertical gradient slab waveguides, each for a different vertical cut, as is shown in Fig. 1. For a given mode number $q$, a set of effective indexes of the vertical gradient slab waveguides form a refractive index profile of a symmetric horizontal slab waveguide. If this slab waveguide has a mode of order $p$, then its value is an approximation of an effective index $n_{p q}$ of the composite, inverted rib structure. Dispersion equations for vertical and horizontal slab waveguides can be derived from the transfer matrix method that provides a straightforward formulation of the multilayer slab waveguide problem. Considering the waveguide isotropic, lossless and composed of $r$ layers, the dispersion equation for TE modes is given by

$$
\left[\begin{array}{c}
E_{x b} \\
\frac{\mathrm{d} E_{x b}}{\mathrm{~d} y}
\end{array}\right]=\prod_{i=1}^{r} M_{i}\left[\begin{array}{c}
E_{x c} \\
\frac{\mathrm{d} E_{x c}}{\mathrm{~d} y}
\end{array}\right]=\left[\begin{array}{ll}
m_{11} & m_{12} \\
m_{21} & m_{22}
\end{array}\right]\left[\begin{array}{c}
E_{x c} \\
\frac{\mathrm{d} E_{x c}}{\mathrm{~d} y}
\end{array}\right]
$$

where $E_{x b}$ and $E_{x c}$ are values of a TE mode primary field component at boundaries of layers adjacent to substrate and ambient (cover) respectively. Dispersion equation for TM modes is given by

$$
\left[\begin{array}{c}
H_{x b} \\
\frac{\mathrm{j}}{\omega \varepsilon_{0} n_{\mathrm{s}}^{2}} \frac{\mathrm{d} H_{x b}}{\mathrm{~d} y}
\end{array}\right]=\prod_{i=1}^{r} M_{i}\left[\begin{array}{c}
H_{x c} \\
\frac{\mathrm{j}}{\omega \varepsilon_{0} n_{\mathrm{c}}^{2}} \frac{\mathrm{d} H_{x c}}{\mathrm{~d} y}
\end{array}\right]
$$

$$
=\left[\begin{array}{ll}
m_{11} & m_{12} \\
m_{21} & m_{22}
\end{array}\right]\left[\begin{array}{c}
E_{x c} \\
\frac{\mathrm{j}}{\omega \varepsilon_{0} n_{\mathrm{c}}^{2}} \frac{\mathrm{d} E_{x c}}{\mathrm{~d} y}
\end{array}\right],
$$

where $H_{x s}$ and $H_{x c}$ are values of a TM mode primary field component at boundaries of layers adjacent to substrate and ambient (cover), respectively, $\mathrm{j}$ is the imaginary unit, $\varepsilon_{0}$ is the free space permitivity and $\omega$ is the angular frequency. The elements $m_{i j}$ for $n_{i} \geq n_{\text {eff }}$ are given by

$$
\left\{\begin{array}{l}
m_{11}=m_{22}=\cos \left(k_{0} d_{i} \sqrt{n_{i}^{2}-n_{\mathrm{eff}}^{2}}\right) \\
m_{12}=\frac{-n_{i}^{2 \rho}}{k_{0} \sqrt{n_{i}^{2}-n_{\text {eff }}^{2}}} \sin \left(k_{0} d_{i} \sqrt{n_{i}^{2}-n_{\mathrm{eff}}^{2}}\right) \\
m_{21}=\frac{k_{0} \sqrt{n_{i}^{2}-n_{\text {eff }}^{2}}}{n_{i}^{2 \rho}} \sin \left(k_{0} d_{i} \sqrt{n_{i}^{2}-n_{\mathrm{eff}}^{2}}\right)
\end{array}\right.
$$

and for $n_{i}<n_{\text {eff }}$ by

$$
\left\{\begin{array}{l}
m_{11}=m_{22}=\cos \left(k_{0} d_{i} \sqrt{n_{\mathrm{eff}}^{2}-n_{i}^{2}}\right) \\
m_{12}=\frac{-n_{i}^{2 \rho}}{k_{0} \sqrt{n_{\mathrm{eff}}^{2}-n_{i}^{2}}} \sinh \left(k_{0} d_{i} \sqrt{n_{\mathrm{eff}}^{2}-n_{i}^{2}}\right) \\
m_{21}=\frac{k_{0} \sqrt{n_{\mathrm{eff}}^{2}-n_{i}^{2}}}{n_{i}^{2 \rho}} \sinh \left(k_{0} d_{i} \sqrt{n_{\mathrm{eff}}^{2}-n_{i}^{2}}\right)
\end{array}\right.
$$

where $n_{i}, d_{i}$ are values of $i$-th layer refractive index and thickness, $k_{0}$ is the vacuum wavenumber, $n_{\text {eff }}$ is an effective refractive index, $\rho=0$ for a TE polarization and $\rho=1$ for a TM polarization.

\section{Analysis results and discussion}

Presented inverted rib waveguide structure is composite if one of the two, or both conditions are met: a thickness of the uniform film is below cut-off, the gradient index channel waveguide is cut off. Refractive indexes of the uniform film and substrate for $\lambda=0.677 \mu \mathrm{m}$ are $n_{2}=1.7775$, and $n_{b}=1.508$. Dispersion equation for a single layered uniform slab [1] allows to determinate cut-off thickness values of subsequent modes. These values for zero-order modes, for two values of ambient refractive index are presented in Table I.

TABLE I

Uniform $\mathrm{SiO}_{2}-\mathrm{TiO}_{2}$ slab cut-off thickness $d_{\text {cut }}[\mu \mathrm{m}]$ for zero-order TE and TM modes.

\begin{tabular}{c|c|c}
\hline \hline & Cover: $n_{\mathrm{c}}=1.00$ & Cover: $n_{\mathrm{c}}=1.33$ \\
\hline $\mathrm{TE}_{0}$ & 0.102 & 0.076 \\
$\mathrm{TM}_{0}$ & 0.152 & 0.109
\end{tabular}

If RIP of the channel waveguide is given by Eq. (2), there are three parameters the cut-off condition is dependent on: $\Delta n, d$, and $e$. An analysis is carried out with an assumption that $\Delta n$ is the only free parameter whereas $d=5 \mu \mathrm{m}$ and $e=0.5$. The parameters describing RIP are: $n_{b}=1.508, \Delta n=0.002, d=5 \mu \mathrm{m}, e=0.5$, $\delta n=10^{-5}$. From dispersion equations cut-off values of maximal refractive index contrast $\Delta n_{\text {cut }}$ were derived. These values for $n_{\mathrm{c}}=1.00$ and 1.33 are presented in Table II.

Taking into account cut-off parameters presented in Tables I and II a variation range of $\Delta n$ is limited to values for which neither of these layers are able to support propagation independently. In Fig. 2 are shown effective index 
TABLE II

Gradient index channel waveguide cut-off maximal refractive index contrast $\Delta n_{\text {cut }}$.

\begin{tabular}{c|c|c}
\hline \hline & Cover: $n_{\mathrm{c}}=1.00$ & Cover: $n_{\mathrm{c}}=1.33$ \\
\hline $\mathrm{TE}_{00}$ & 0.0022 & 0.0022 \\
$\mathrm{TM}_{00}$ & 0.0022 & 0.0022 \\
$\mathrm{TE}_{01}$ & 0.0201 & 0.0194 \\
$\mathrm{TM}_{01}$ & 0.0213 & 0.020 \\
$\mathrm{TE}_{10}$ & 0.0425 & 0.0411 \\
$\mathrm{TM}_{10}$ & 0.0444 & 0.0424
\end{tabular}

TABLE III

Critical thickness $d_{2 \mathrm{~g}}$ of the uniform $\mathrm{SiO}_{2}-\mathrm{TiO}_{2}$ film above which modes are confined to this film.

\begin{tabular}{c|c|c}
\hline \hline & Cover: $n_{\mathrm{c}}=1.00$ & Cover: $n_{\mathrm{c}}=1.33$ \\
\hline $\mathrm{TE}_{00}$ & 0.110 & 0.084 \\
$\mathrm{TM}_{00}$ & 0.163 & 0.120 \\
$\mathrm{TE}_{10}$ & 0.117 & 0.091 \\
$\mathrm{TM}_{10}$ & 0.173 & 0.129 \\
$\mathrm{TE}_{20}$ & 0.125 & 0.099 \\
$\mathrm{TM}_{20}$ & 0.183 & 0.141
\end{tabular}

characteristics of the inverted rib waveguide in function of $d_{2}$ for $n_{\mathrm{c}}=1.33$. The assumption was taken that $\Delta n=0.002$ which is below the cut-off for both fundamental modes. The curves for $\mathrm{TE}_{20}-\mathrm{TE}_{50}, \mathrm{TM}_{20}-\mathrm{TM}_{40}$ are not shown because in the presented range of $d_{2}$ these modes are very close to cut-off. For each characteristic $n_{\text {eff }}\left(d_{2}\right)$ there is a characteristic critical value $d_{2 \mathrm{~g}}$ above which a given mode is confined to the slab film. These values for $n_{\mathrm{c}}=1.00$ and 1.33 are presented in Table III.

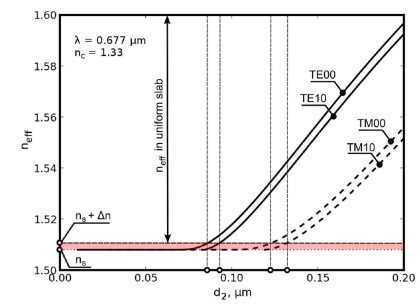

Fig. 2. Effective index characteristics of the inverted rib composite waveguide in function of the $\mathrm{SiO}_{2}-\mathrm{TiO}_{2}$ film thickness $d_{2}$, calculated for $n_{\mathrm{c}}=1.33$.

As can be seen from comparison of values in Tables I and III and from Fig. 2, a presence of the channel waveguide in the substrate downshifted cut-off thickness values. Critical thicknesses $d_{2 \mathrm{~g}}$ are higher than $d_{\text {cut }}$ of the slab waveguide. Each value $n_{\text {c }}$ corresponds with range $d_{2}$ values in which effective index of $\mathrm{TM}_{00}$ mode is slowly varying function of $d_{2}$ limited by $\Delta n$ of RIP in the substrate, whereas a sharp increase is observed for $\mathrm{TE}_{00}$ effective index. In these ranges high values of fundamental mode sensitivity difference are expected.

Homogeneous and surface sensitivities of the channel gradient index waveguides in function of $\Delta n$ can be calculated using Eq. (1). These characteristics are monoton-

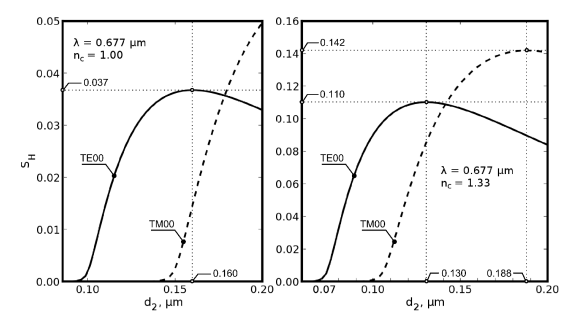

Fig. 3. Homogeneous sensitivity characteristics of the inverted rib waveguide in function of a uniform film thickness $d_{2}$.

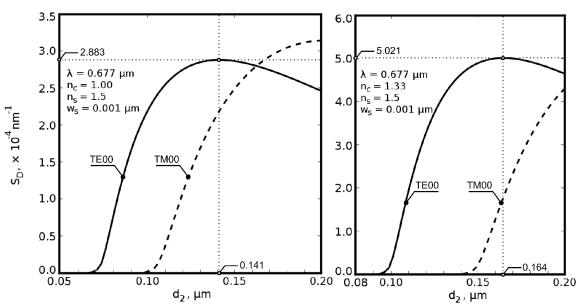

Fig. 4. As in Fig. 3 but for surface sensitivity.

ically increasing functions. Values of both homogeneous and surface sensitivities are small which is typical for ion-exchange based gradient index waveguides [12, 13]. In Figs. 3 and 4 are shown sensitivity characteristics of the inverted rib waveguide, whereas Fig. 5 presents homogeneous and surface sensitivity difference characteristics for $\mathrm{TE}_{00}-\mathrm{TM}_{00}$ modes. In both cases sensitivities of $\mathrm{TM}_{00}$ mode were subtracted from values of $\mathrm{TE}_{00}$ modes: $\Delta S=S_{\mathrm{TE}}-S_{\mathrm{TM}}$. These characteristics are in function of uniform film thickness $d_{2}$. Characteristics of surface sensitivity were calculated with an assumption that the refractive index and the thickness of the sensitive film are: $n_{\mathrm{s}}=1.5$ and $w_{\mathrm{s}}=1 \mathrm{~nm}$. As it can be seen, comparing these characteristics with the ones in Fig. 2, a presence of high refractive index uniform film substantially increases sensitivity even though the channel waveguide itself is below cut-off. Each sensitivity characteristic has a single local maximum. Maxima for $\mathrm{TM}_{00}$ outreach the range of $d_{2}$ presented in Figs. 3 and 4 in all cases but one: $S_{\mathrm{H}}$ for $n_{\mathrm{c}}=1.00$.

From Fig. 4 it can be seen that an increase off ambient refractive index causes the downshift of uniform film thickness values for which mode homogeneous sensitivity of $\mathrm{TE}_{00}$ is maximal. This thickness is less than $d_{2 \mathrm{~g}}$ of $\mathrm{TM}_{00}$ mode when $n_{\mathrm{c}}=1.00$ and higher than $d_{2 \mathrm{~g}}$ when $n_{\mathrm{c}}=1.33$. Therefore for higher values of ambient refractive index the homogeneous sensitivity of $\mathrm{TE}_{00}$ mode is maximal when both fundamental modes are confined to the uniform film. Homogeneous sensitivity values of presented structure are comparable with the ones for a $\mathrm{SiO}_{2}-\mathrm{TiO}_{2}$ rib waveguides that are several hundred nm thick and therefore not compatible with optical fibres [17]. Similar change of relationship between the uniform film thickness maximizing $S_{H}$ of $\mathrm{TE}_{00}$ mode and 
critical thickness of $\mathrm{TM}_{00}$ mode is observed for surface sensitivity characteristics.
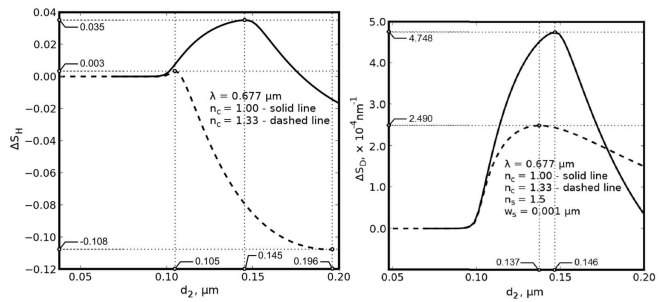

Fig. 5. Homogeneous and surface sensitivity difference characteristics of $\mathrm{TE}_{00}-\mathrm{TM}_{00}$ modes of the inverted rib waveguide in function of a uniform film thickness $d_{2}$.

Each of the sensitivity difference characteristics that are shown in Fig. 5 has two local opposite extremes. In contradiction to three-layered slab waveguide, first extreme on homogeneous sensitivity difference characteristics is achieved when sensitivity of $\mathrm{TE}_{00}$ mode is higher than the one of $\mathrm{TM}_{00}$ mode $\left(d_{2 m}=0.105 \mu \mathrm{m}\right.$ and $0.145 \mu \mathrm{m})$. These values are smaller than critical thicknesses $d_{2 \mathrm{~g}}$ of $\mathrm{TM}_{00}$ mode for both cover refractive indexes. Therefore for them the structure is composite. The following local minima on both homogeneous and surface sensitivity difference characteristics are achieved when the fundamental modes are confined to a uniform film. In this case the structure should be considered a slab waveguide based on the non-uniform substrate and homogeneous sensitivity of the TM mode is greater than of the TE as is for three-layered slab. In a case of the surface sensitivity difference characteristics, only the characteristic for $n_{\mathrm{c}}=1.00$ has a maximum for the uniform film thickness less than critical of a $\mathrm{TM}_{00}$ mode $\left(d_{2 m}=0.146 \mu \mathrm{m}\right)$, therefore for $n_{\mathrm{c}}=1.33$ presented structure is a slab waveguide $\left(d_{2 m}=0.137 \mu \mathrm{m}\right)$. This can be explained alleging a fact that a magnitude of changes of effective indexes is preponderantly determined by refractive index changes at the interface of the uniform film, where is the thin sensitive film of relatively high refractive index comparable with substrate refractive index $n_{b}$.

This work presents a theoretical analysis of the relation between selected parameters of inverted rib waveguide structure and its effective index and sensitivity characteristics. Considering that the maximal refractive index contrast $\Delta n$ of gradient channel waveguide is less than cut-off, an additional layer is required to make light guidance possible. A presence of thin uniform, high refractive index film on a top of this waveguide was investigated. It was shown that for given ambient refractive index one can find a range of uniform film thickness values in which $\mathrm{TE}_{00}$ mode is confined to the uniform film whereas $\mathrm{TM}_{00}$ mode is guided in both: the channel waveguide and the uniform film. In these case the presented structure is composite. Homogeneous and surface sensitivity characteristics have single local maximum obtained for given uniform film thickness. It was shown that for the assumpted uniform film refractive index only for $n_{\mathrm{c}}=1.00$ this thickness is less than critical. Considering the difference interference of fundamental modes a homogeneous sensitivity difference is maximized when thickness of the uniform film is less than the critical one for both considered cover refractive indexes: $n_{\mathrm{c}}=1.00$ and 1.33 whereas the surface sensitivity is maximized within desired range of uniform film thickness only for $n_{\mathrm{c}}=1.00$. For higher ambient refractive index the surface sensitivity is maximal if both fundamental modes are confined to the uniform film because of high value of sensitivity film refractive index $n_{\mathrm{s}}=1.5$, despite of its thickness $d_{\mathrm{s}}=1 \mathrm{~nm}$ that is several hundred times less than a wavelength $\lambda=0.677 \mu \mathrm{m}$. Further analysis must involve a refractive index of the uniform film as a second parameter. Diminution of this value should allow finding range of uniform film parameters within which the homogeneous sensitivity and surface sensitivity difference of fundamental modes is maximal for uniform film thickness less than a critical thickness of $\mathrm{TM}_{00}$ mode.

\section{Acknowledgments}

This work is sponsored by Polish Ministry of Science and Higher Education within a scope of the grant DEC$-2011 / 01 /$ B/ST7/06525.

\section{References}

[1] W. Lukosz, Sensors Actuat. B 29, 37 (1995).

[2] P. Karasiński, Opto-Electron. Rev. 15, 168 (2007).

[3] P. Karasiński, Opto-Electron. Rev. 19, 10 (2011).

[4] Z. Qi, S. Zhao, F. Chen, R. Liu, S. Xia, Opt. Expr. 18, 7421 (2010).

[5] P. Karasiński, C. Tyszkiewicz, R. Rogoziński, Opt. Appl. 41, 351 (2011).

[6] M. Wang, J. Hiltunen, C. Liedert, S. Pearce, M. Charlton, L. Hakalahti, P. Karioja, R. Myllylä, Opt. Expr. 20, 20309 (2012).

[7] P. Karasiński, C. Tyszkiewicz, R. Rogoziński, J. Jaglarz, J. Mazur, Thin Solid Films 519, 5544 (2011).

[8] K. Schmitt, K. Oehse, G. Sulz, Ch. Hoffmann, Sensors 8, 711 (2008).

[9] C. Tyszkiewicz, Acta Phys. Pol. A 122, 908 (2012).

[10] R. Bruck, E. Melnik, P. Muellner, R. Hainberger, M. Lämmerhofer, Biosens. Bioelectron. 26, 3832 (2011).

[11] M. Wang, J. Hiltunen, S. Uusitalo, J. Puustinen, J. Lappalainen, P. Karioja, R. Myllylä, J. Microelectron. Eng. 88, 175 (2011).

[12] R. Rogoziński, P. Karasiński, Opto-Electron. Rev. 13, 229 (2005).

[13] P. Karasiński, Opto-Electron. Rev. 19, 1 (2011).

[14] R. Rogoziński, in: Ion Exchange in Glass - The Changes of Glass Refraction, Ch. 7, Ed. A. Kilislioğlu, InTech, Rijeka 2012, p. 155.

[15] K.S. Chiang, Proc. SPIE 2399, 2 (1995).

[16] J. Chilwell, I. Hodgkinson, J. Opt. Soc. Am. A 1(7), 742 (1984).

[17] C. Tyszkiewicz, Opt. Appl. 42, 555 (2012). 